\title{
Phase shifts of synchronized oscillators and the systolic-diastolic blood pressure relation
}

\author{
L. Angelini, ${ }^{1,2,3}$ G. Lattanzi, ${ }^{4}$ R. Maestri, ${ }^{5}$ D. Marinazzo, ${ }^{1,2}$ G. Nardulli, ${ }^{1,2,3}$ L. Nitti, ${ }^{1,3,6}$ M. Pellicoro, ${ }^{1,2,3}$ \\ G. D. Pinna, ${ }^{5}$ and S. Stramaglia ${ }^{1,2,3}$ \\ ${ }^{1}$ TIRES-Center of Innovative Technologies for Signal Detection and Processing, Università di Bari, Bari, Italy \\ ${ }^{2}$ Dipartimento Interateneo di Fisica, Bari, Italy \\ ${ }^{3}$ Istituto Nazionale di Fisica Nucleare, Sezione di Bari, Bari, Italy \\ ${ }^{4}$ Abt. Theoretische Physik SF5, Hahn-Meitner Institut, Glienickerstrasse 100, 14109 Berlin, Germany \\ ${ }^{5}$ Divisione di Cardiologia e Bioingegneria, Fondazione Salvatore Maugeri, IRCCS Istituto Scientifico di Montescano (PV), Italy \\ ${ }^{6}$ D.E.T.O., University of Bari, Bari, Italy \\ (Received 18 February 2004; published 23 June 2004)
}

\begin{abstract}
We study the phase-synchronization properties of systolic and diastolic arterial pressure in healthy subjects. We find that delays in the oscillatory components of the time series depend on the frequency bands that are considered, in particular we find a change of sign in the phase shift going from the very low frequency band to the high frequency band. This behavior should reflect a collective behavior of a system of nonlinear interacting elementary oscillators. We prove that some models describing such systems, e.g., the Winfree and the Kuramoto models, offer a clue to this phenomenon. For these theoretical models there is a linear relationship between phase shifts and the difference of natural frequencies of oscillators and a change of sign in the phase shift naturally emerges.
\end{abstract}

DOI: 10.1103/PhysRevE.69.061923

PACS number(s): 87.19.Uv, 05.10.-a, 05.45.-a

\section{INTRODUCTION}

Time series of physiological origin very often display synchronous behavior. Most likely, this is the result of collective behavior of a huge number of nonlinearly interacting elementary oscillators. Different examples of this phenomenon, as well as models of it, can be found, for example, in [1]. In the present paper we address a further application dealing with the dynamics of arterial blood pressure waves in humans. Systolic blood pressure (SAP) is the (maximal) pressure within the cardiovascular system as the heart pumps blood into the arteries. Diastolic pressure (DAP) represents the minimum in pressure, as happens while the heart rests and fills with blood, prior to the next contraction; we investigate the relation between DAP and SAP signals in healthy subjects. Blood pressure fluctuations represent the complex interplay between several modulating factors like heart rate, peripheral resistance, cardiac contractility, central venous pressure, and respiration. Since all these factors are under the control of the neural mechanisms responsible for cardiovascular and respiratory regulation, the analysis of these fluctuations may provide significant information on the physiology and pathophysiology of the autonomic control of the cardiovascular function [2,3]. Synchronization of SAP and DAP is expected, though a detailed study of its features is apparently still lacking. In a previous paper [4] it was noticed that DAP and SAP are characterized by a phase lag in the very low frequency band (VLF). The analysis of [4] uses Fourier analysis, which is not particularly useful when nonstationary effects play a relevant role. In the present work we address two questions about the DAP/SAP relationship: Is the phase lag depending on the frequency band? Is the phase lag connected to a causal relation between SAP and DAP? To address these questions, we measured the DAP and SAP signals in a number of healthy subjects. Studying the mutual interdependency between the two time series, we conclude that there is not a causal relationship between DAP and SAP time series, i.e., neither of the two is the driver for the other. Moreover, a significant phase delay is found, for healthy subjects, in the VLF band and in the high frequency (HF) band. The phase shift between DAP and SAP is positive in the VLF band and negative in the HF band. This change of sign in the phase shift has its origin in the regulatory mechanisms of blood circulation. A physiological interpretation of these mechanisms is beyond the scope of our work; however, the hypothesis that synchronization results from the collective behavior of elementary nonlinear oscillators may offer a clue to its understanding. To exploit this idea we use below two well-known models of coupled oscillators, the Winfree model [5] and the Kuramoto model [6].

Winfree's paper [5] on coupled oscillators provided one of the first tractable examples of a self-organizing system. Winfree introduced an approximation that has become the standard approach to the study of ensembles of biological oscillators: In the weak coupling limit, amplitude variations could be neglected and the oscillators could be described only by their phases along their limit cycles. Winfree also discovered that systems of oscillators with randomly distributed frequencies exhibit a remarkable cooperative phenomenon, reminiscent of a thermodynamic phase transition, as the variance of the frequencies is reduced. The oscillators remain incoherent, each running near its natural frequency, until a certain threshold is crossed. Then the oscillators begin to synchronize spontaneously. The Winfree model was subsequently modified by Kuramoto who provided an analytically solvable version of it [6]. This field of study has been very active all along and the analysis of synchronization phenomena remains a thriving area of research, see [7] for a review. Having in mind our experimental findings on the SAP/DAP relation, we first examine in Sec. II the phase shift between coupled oscillators in these models, once synchronization has been reached. We observe that there exists a simple lin- 
ear dependence between phase shifts of synchronized oscillators and the difference between their natural frequencies. This phenomenon offers a simple mechanism to describe the change of sign in the phase lag as the frequency band is changed. In Sec. III we describe the experimental data and analyze them using the theoretical approach of Sec. II. Moreover, we discuss the problem of the causal relation between the DAP/SAP time series. Finally in Sec. IV we draw our conclusions.

\section{PHASE SHIFTS OF SYNCHRONIZED OSCILLATORS}

\section{A. Winfree model}

The Winfree model is defined by the set of equations $(i$ $=1, \ldots, N)$

$$
\dot{\theta}_{i}(t)=\omega_{i}+\frac{1}{N} \sum_{j=1}^{N} \kappa P\left(\theta_{j}\right) R\left(\theta_{i}\right) .
$$

It describes a set of $N \gg 1$ coupled nonlinear oscillators, with coupling constant proportional to $\kappa \cdot \theta_{i}(t)$ is the phase of the $i$ th oscillator and $\left\{\omega_{i}\right\}$ describes a set of natural frequencies taken randomly from a distribution $g(\omega) . P\left(\theta_{j}\right)$ is the influence function of the $j$ th oscillator and $R\left(\theta_{i}\right)$ is the sensitivity function giving the response of the $i$ th oscillator. We shall assume below $g(\omega)=1 / 2 \gamma$ for $\gamma \in\left[\omega_{0}-\gamma, \omega_{0}+\gamma\right], g(\omega)=0$ otherwise. In the previous equation $P(\theta)=1+\cos \theta, R(\theta)=$ $-\sin \theta$. The phase diagram of the Winfree model has been recently discussed [8]. In particular the long-time behavior of the system is characterized by a synchronous dynamics for $\kappa$ and $\gamma$ not very large. For $\omega_{0}=1$ synchronization occurs for $\kappa<0.77$ and $\gamma<h(\kappa)$, where the function $h(\kappa)$ can be found in Fig. 3 of Ref. [8]; in any case $\gamma<0.2$. This means that all the oscillators are characterized by a common average frequency (or rotation number) $\rho_{i}=\lim _{t \rightarrow \infty} \theta_{i}(t) / t$. The Winfree model can describe different sets of pulse-coupled biological oscillators, see, e.g., [9-11].

We now wish to study the relation between the phase shift $\delta \theta$ of a pair of oscillators and the difference of their natural frequencies $\delta \omega$. We have performed numerical simulations with $N=500$ oscillators with different values of $\kappa$ and $\gamma$ $=0.10$ corresponding to the synchronization phase. We have considered times as large as $t=1000$. As expected there is no dependence on the initial conditions. On the contrary $\delta \theta$ is linearly related to $\delta \omega$ as shown in Fig. 1, where we plot $\rho_{i}$ versus $\omega_{i}$ for various values of $\kappa$ (on the left) and $\delta \theta$ versus $\delta \omega$ (on the right). This dependence can be understood as follows. As $N \rightarrow \infty$, the sum over all oscillators in Eq. (1) can be replaced by an integral, yielding

$$
v(\theta, t, \omega)=\omega-\sigma(t) \sin \theta,
$$

where

$$
\sigma(t)=\kappa \int_{0}^{2 \pi} \int_{1-\gamma}^{1+\gamma}(1+\cos \theta) p(\theta, t, \omega) g(\omega) d \omega d \theta .
$$

Here $p(\theta, t, \omega)$ denotes the density of oscillators with phase $\theta$ at time $t$. We consider the large $t$ behavior to allow for syn-
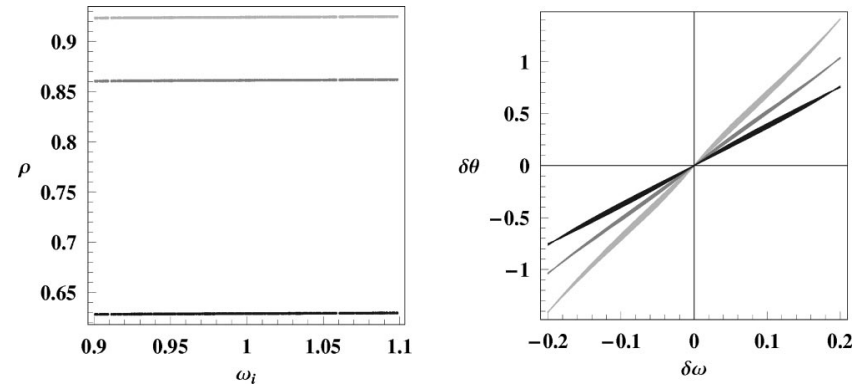

FIG. 1. Left: the rotation number $\rho$ plotted versus $\omega$ for $\gamma$ $=0.10$ and $\kappa=0.35,0.45,0.65$ (from top to bottom). Right: $\delta \theta$ vs $\delta \omega$ for the same values of $\gamma$ and $\kappa$ (larger slopes correspond to smaller values of $\kappa$ ).

chronization; moreover, we take a temporal averaging over the common period $T$ to get rid of local fluctuations. We get

$$
v=\omega-\frac{1}{T} \int_{t}^{t+T} d t \sigma(t) \sin \theta(t)
$$

and consider variations in $\omega$ :

$$
0=\delta \omega-\frac{1}{T} \int_{t}^{t+T} d t \sigma(t) \delta \theta(t) \cos \theta(t)
$$

Since the oscillators are synchronized $\delta \theta(t)$ is timeindependent for a large enough $t$. Therefore,

$$
\begin{aligned}
\delta \omega= & \frac{\kappa \delta \theta}{T} \int_{t}^{t+T} d t \int d \omega g(\omega) \int_{0}^{2 \pi} d \hat{\theta}(1 \\
& +\cos \hat{\theta}) p(\hat{\theta}, t, \omega) \cos \theta(t) \\
= & \frac{\kappa \delta \theta}{T} \int_{t}^{t+T} d t \cos \theta(t)+\frac{\kappa \delta \theta}{2 T} \int_{t}^{t+T} d t \\
& \times \int d \omega g(\omega) \int_{0}^{2 \pi} d \hat{\theta} p(\hat{\theta}, t, \omega) \cos [\hat{\theta}+\theta(t)]+\frac{\kappa \delta \theta}{2 T} \int_{t}^{t+T} \\
& \times d t \int d \omega g(\omega) \int_{0}^{2 \pi} d \hat{\theta} p(\hat{\theta}, t, \omega) \cos [\hat{\theta}-\theta(t)] .
\end{aligned}
$$

The first two terms on the right-hand side of Eq. (6) vanish since the integrand functions have zero temporal average. We get, therefore,

$$
\delta \omega=\frac{\kappa \lambda}{2} \delta \theta
$$

which is the desired linear relation between $\delta \omega$ and $\delta \theta$. The factor $\lambda$ is evaluated as follows: 

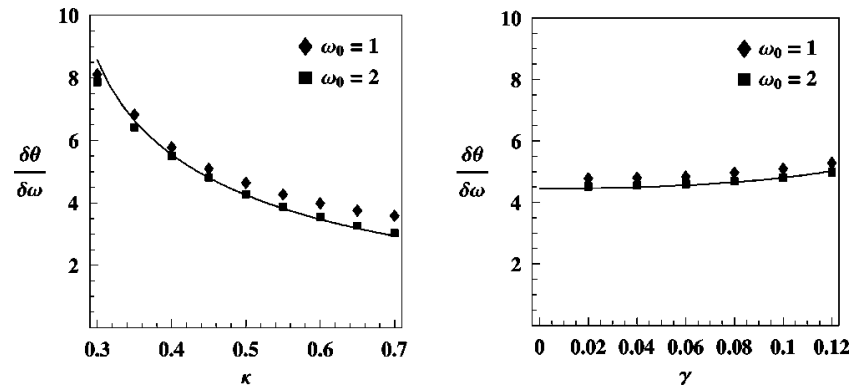

FIG. 2. The slope $\delta \theta / \delta \omega$ in the Winfree model. Left: $\delta \theta / \delta \omega$ as a function of $\kappa$ for two values of $\omega_{0}$ and $\gamma=0.1$. Right: $\delta \theta / \delta \omega$ as a function of $\gamma$ for two values of $\omega_{0}$ and $\kappa=0.45$. The curves are independent of $\omega_{0}$.

$$
\begin{aligned}
\lambda & =\frac{1}{T} \int_{t}^{t+T} d t \int_{1-\gamma}^{1+\gamma} d \omega g(\omega) \int_{0}^{2 \pi} d \hat{\theta} p(\hat{\theta}, t, \omega) \cos [\hat{\theta}-\theta(t)] \\
& =\frac{1}{N} \sum_{j=1}^{N} \frac{1}{T} \int_{t}^{t+T} d t \cos \left[\theta_{j}(t)-\theta(t)\right]=\frac{1}{N} \sum_{j=1}^{N} \cos \left[\delta \theta_{j}\right] \\
& =\frac{4 \gamma}{\kappa \lambda} \int_{-1}^{+1} d y \widetilde{g}\left(\frac{4 \gamma y}{\kappa \lambda}\right) \cos \left(\frac{4 \gamma y}{\kappa \lambda}\right) .
\end{aligned}
$$

Here $\tilde{g}(\delta \theta)$ is a probability distribution of $\delta \theta$. It is related to the probability distribution of $\delta \omega$ by Eq. (7). Both the $\delta \omega$ and the $\delta \theta$ distribution functions are derived from the $\omega$ density $g(\omega)$. If this density is flat, as assumed here, then $\tilde{g}$ $=g * g$, i.e.,

$$
\widetilde{g}(\delta \theta)=\frac{\kappa \lambda}{4 \gamma}-\left(\frac{\kappa \lambda}{4 \gamma}\right)^{2}|\delta \theta| .
$$

In conclusion $\lambda$ is given by solving the equation

$$
\frac{4 \gamma^{2}}{\kappa^{2}}=\lambda \sin ^{2}\left(\frac{2 \gamma}{\kappa \lambda}\right)
$$

We notice that in Eq. (7) there is no dependence on $\omega_{0}$; this dependence is in the first two terms of Eq. (6) since they vanish only in the large $N$, large $t$ limit.

In Fig. 2 we report the slope $\delta \theta / \delta \omega$ as computed by Eq. (10) as a function of $\kappa$ (with $\gamma=0.1$ ) on the left and as a function of $\gamma$ (with $\kappa=0.45)$ on the right. This curve is independent of $\omega_{0}$. We also report results of the numerical analysis for two values of $\omega_{0}$. These data show a small dependence on $\omega_{0}[12]$.

\section{B. Kuramoto model}

The analysis of the Kuramoto model produces comparable results. The Kuramoto model is based on the set of equations $(i=1, \ldots, N)$

$$
\dot{\theta}_{i}(t)=\omega_{i}+\frac{\kappa}{N} \sum_{j=1}^{N} \sin \left(\theta_{i}-\theta_{j}\right) .
$$

The numerical results one gets are similar to those of Fig. 1, with a linear dependence of $\delta \theta$ on $\delta \omega$ and the rotational
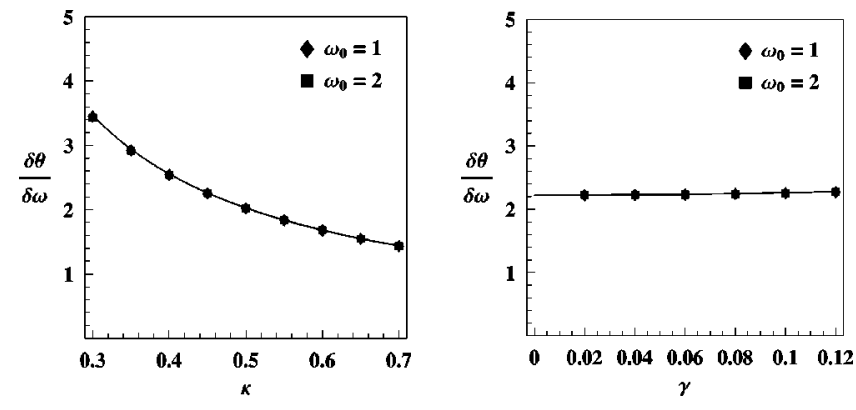

FIG. 3. The slope $\delta \theta / \delta \omega$ in the Kuramoto model. Left: $\delta \theta / \delta \omega$ as a function of $\kappa$ for two values of $\omega_{0}$ and $\gamma=0.1$. Right: $\delta \theta / \delta \omega$ as a function of $\gamma$ for two values of $\omega_{0}$ and $\kappa=0.45$.

frequency $\rho_{i}=\omega_{0}$ (we use the same distribution function $g(\omega)$ as before). The latter results follow from the fact that the phases $\theta_{i}$ are dynamically pulled toward the mean phase [7]. These results can be compared with an analytical treatment by observing that, in this case, instead of Eq. (6) one gets

$$
\delta \omega=\frac{\kappa \delta \theta}{T} \int_{t}^{t+T} d t \int d \omega g(\omega) \int_{0}^{2 \pi} d \hat{\theta} p(\hat{\theta}, t, \omega) \cos [\hat{\theta}-\theta(t)]
$$

Due to the absence of terms analogous to the first and second terms on the right-hand side of Eq. (6), we expect a better agreement between numerical simulations and analytical evaluation. From Eq. (12) we get, instead of Eq. (7):

$$
\delta \omega=\kappa \lambda \delta \theta,
$$

with $\lambda$ given by

$$
\frac{2 \gamma^{2}}{\kappa^{2}}=\lambda\left(1-\cos \frac{2 \gamma}{\kappa \lambda}\right) \text {. }
$$

These results are reported in Fig. 3 together with the results of the numerical simulations.

\section{SYSTOLIC/DIASTOLIC ARTERIAL PRESSURE RELATION}

\section{A. Phase shifts from arterial pressure data}

Let us consider two time series: $x_{S}(t)$ and $x_{D}(t)$, representing systolic and diastolic arterial pressure. Data are from a population of 47 normal subjects (mean age $+/-$ standard deviation: $54+/-8$ years) who underwent a 10 min supine resting recording of ECG and noninvasive arterial blood pressure (by the Finapres device), in the laboratory for the assessment of Autonomic Nervous System, S. Maugeri Foundation, Scientific Institute of Montescano, Italy. For each cardiac cycle, corresponding values of SAP and DAP were computed and resampled at a frequency of $2 \mathrm{~Hz}$ using a cubic spline interpolation. In Fig. 4 we report the time series of the systolic arterial pressure $x_{S}(t)$ for one of the subjects examined in this study.

These data are analyzed by filtering in appropriate frequency bands. We consider here three bands: very low frequency (VLF) band: $(0.01,0.04) \mathrm{Hz}$; low frequency (LF) 

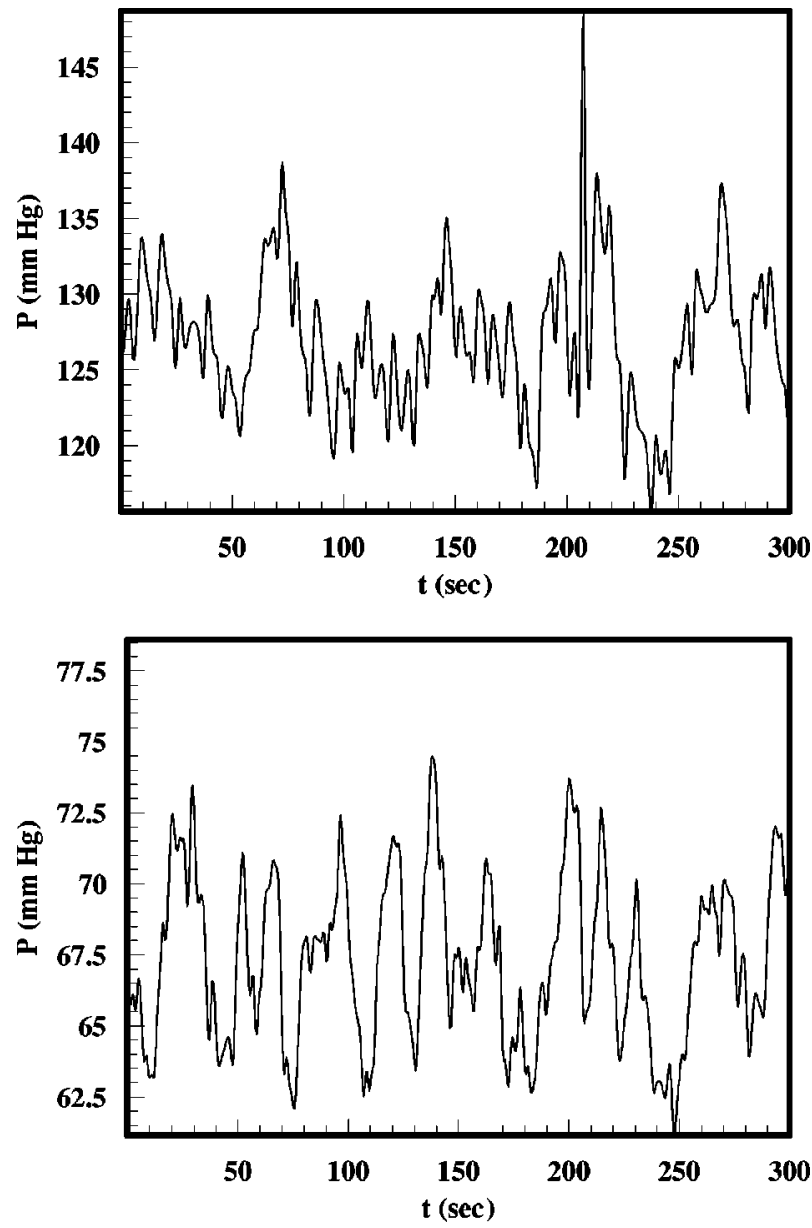

FIG. 4. The time series $x_{S}(t)$ (left) and $x_{D}(t)$ (right) of the systolic and diastolic arterial pressures for one of the subjects examined.

band: $(0.04,0.15) \mathrm{Hz}$; and high frequency (HF) band: $(0.15,0.45) \mathrm{Hz}$. In a previous paper [4], using Fourier transform methods, occurrence of delays between SAP and DAP was investigated, and it was found that DAP anticipates SAP in VLF (delay equal to $2.5 \mathrm{~s}$ ) and in LF ( $0.6 \mathrm{~s})$; no significant delay was found in HF. Here we enlarge the statistical population with respect to [4] and evaluate the phases of signals by the analytic signal technique, which allows a better estimate. As is well known SAP and DAP are highly synchronized and our data confirm this expectation. We have used the Hilbert transform method that allows one to detect phase synchronization in noisy scalar signals [13]. To extract a phase from the signal one considers the Hilbert transform of the original time series

$$
y(t)=\frac{1}{\pi} \mathrm{P} \int_{-\infty}^{+\infty} \frac{x(\tau)}{t-\tau} d \tau,
$$

where $\mathrm{P}$ denotes Cauchy principal value. Then one forms the analytic signal $z(t)=x(t)+i y(t)=A(t) e^{i \phi(t)}$, where $A(t)$ $=\sqrt{x^{2}(t)+y^{2}(t)}$ and $\phi(t)$ is the desired phase. To control the possible synchronization of two signals $x_{1}(t), x_{2}(t)$ the following procedure is applied: the phases $\phi_{1}(t)$ and $\phi_{2}(t)$ are
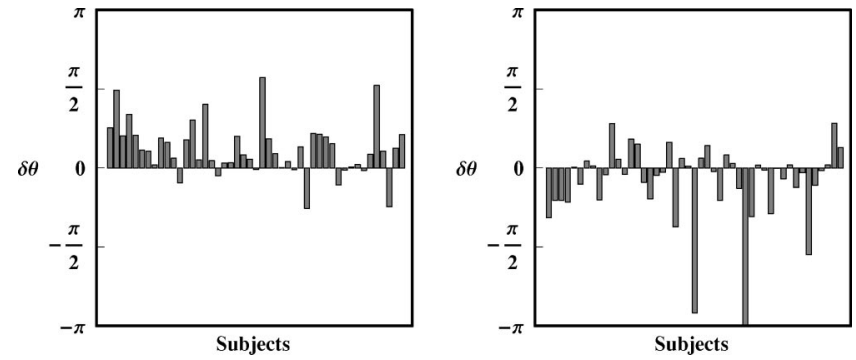

FIG. 5. The phase shifts $\delta \theta$ for all 47 subjects filtered in the VLF (left) and HF (right) bands.

computed and the so-called generalized phase differences $\Phi_{n, m}(t)=\left[m \phi_{1}(t)-n \phi_{2}(t)\right]_{\bmod 2 \pi}$, with $n, m$ integers, are evaluated. In the present study only $1: 1$ synchronization has been examined and the two phases $\phi_{1}(t), \phi_{2}(t)$ coincide with the phases of the time series $x_{D}(t), x_{S}(t)$. Phase synchronization is characterized by the appearance of peaks in the distribution of the phase difference. To evaluate the phase shift we have considered time intervals characterized by a constant phase difference between the two series:

$$
\delta \theta=\theta_{D}(t)-\theta_{S}(t) .
$$

We find $\delta \theta>0$ in the VLF band, i.e., in this band diastolic pressure anticipates systolic pressure. Our analysis confirms the results of [4] with a different method. On the other hand in the HF band (in basal conditions) the phase shift is negative $\delta \theta<0$, which means that in this band the systolic pressure signal anticipates the diastolic one. These data are reported for all 47 subjects in Fig. 5 that shows on the left the VLF band and on the right the HF band (we have not reported data in the intermediate region LF band, as they are compatible with $\delta \theta=0$ ). We estimated $1.76 \times 10^{-6}$ to be the probability that the phase shifts in the VLF band are sampled from a distribution whose mean is less than or equal to zero; analogously $3.0 \times 10^{-2}$ is the probability that the phase shifts in the HF band are sampled from a distribution whose mean is greater than or equal to zero.

On a physiological basis these results mean that the different sets of oscillators producing the time series have different spectral properties. Leaving aside the task of a physiologically based modelization we now show that the results obtained in Sec. II can shed light on this phenomenon. For the present application we use the Winfree model.

\section{B. Interpretation of phase shifts between related oscillatory signals}

We present here a schematic view of phase shifts $\delta \theta$ between the time series $x_{S}(t)$ and $x_{D}(t)$. This picture is only qualitative and aims to reproduce the dependence of the sign of $\delta \theta$ on the filter in the frequency power spectrum. As such, the picture is not realistic and does not offer a physiologically based model of the time series; nevertheless it can shed light on oscillator dynamics underlying them. Let us assume that the two oscillatory signals $x_{S}(t)$ and $x_{D}(t)$ are the result of the collective behavior of two sets of oscillators, sets SAP and DAP, respectively. We assume that this collective behav- 


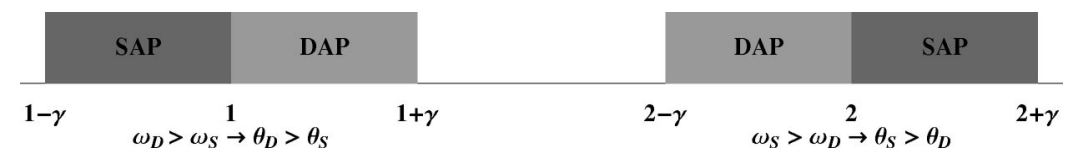

FIG. 6. The two intervals of natural frequencies $A$ and $B$. $A$ is on the left and is centered around the value $a=1 ; B$, on the right, is centered around $b=2$. We assume that oscillators with frequencies in the band SAP (DAP) produce collectively the signal $x_{S}(t)\left(x_{D}(t)\right)$, see the text.

ior produces a systolic arterial pressure (SAP) and diastolic arterial pressure (DAP) time series. We assume that the oscillators in the set SAP have natural frequencies in the domain $\omega \in(a-\gamma, a) \cup(b, b+\gamma)$, while frequencies for the set DAP are in the domain $\omega \in(a, a+\gamma) \cup(b-\gamma, b)$. We also assume $\gamma \leqslant 1$. We will use as numerical values $a=1, b=2$, and $\gamma=0.1$, see Fig. 6 .

On the other hand the two bands $A: 1-\gamma<\omega<1+\gamma$, and $B: 2-\gamma<\omega<2+\gamma$ would model the VLF and HF frequency bands.

Let us assume that the coupling among the oscillators having natural frequencies in the intervals $A$ and $B$ is modeled by the Winfree model, i.e., by Eq. (1). However, we assume for the coupling

$$
\kappa \rightarrow \kappa_{i j}=\kappa H\left[2 \gamma-\left|\omega_{i}-\omega_{j}\right|\right],
$$

where $H$ is the Heaviside function. By this choice there is no interaction between oscillators in the two bands, though a weak coupling would not alter the qualitative picture. We consider one value of $\kappa(\kappa=0.65$ in this case $)$. The two sets of oscillators, one centered around the natural frequency $\omega_{0}=1$ (set $A$ ) and the other around $\omega_{0}=2$ (set $B$ ) become synchronized around two synchronization frequencies, $\omega_{\mathrm{VLF}}=0.62 \mathrm{~Hz}$ and $\omega_{\mathrm{HF}}=1.88 \mathrm{~Hz}$, see Fig. 7 (left side). Correspondingly, on the right, we have two lines showing a linear dependence between $\delta \omega$ and $\delta \theta$. The two lines are almost completely superimposed, which shows a weak dependence on the average natural frequencies of the two sets. The interesting result, however, is related to our definition of VLF and
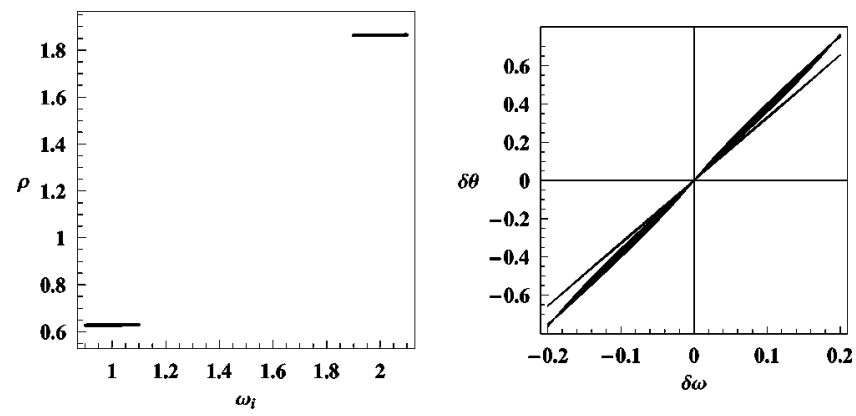

FIG. 7. Left: The oscillators of set $A$, with frequencies centered around $a=1$, become synchronized with a frequency around $\omega_{\mathrm{VLF}}$ $=0.62 \mathrm{~Hz}$; those of set $B$ (frequencies around $b=2$ ) have a synchronization frequency $\omega_{\mathrm{HF}}=1.88 \mathrm{~Hz}$. Right: The phase shift $\delta \theta$ between any pair of oscillators as a function of the difference $\delta \omega$ between the natural frequencies of the oscillators in the pairs. The partially overlapping lines refer to the two sets of oscillators $A$ and $B$, which shows a weak dependence of the slope on the natural frequencies. Numerical results refer to $N=1000$ oscillators, with $\kappa$ $=0.65$.
HF bands. The VLF band is the result of the collective behavior of oscillators in set $A$. For them $\omega_{D}>\omega_{S}$ and therefore, on the basis of the results of Sec. II, $\delta \theta=\theta_{D}(t)$ $-\theta_{S}(t)>0$. On the other hand in the HF band, $\omega_{S}>\omega_{D}$ and therefore $\delta \theta<0$. This simple mechanism implies the effect of a change of sign between the two phases when one goes from the very low frequency to the high frequency.

One might wonder if our data also show a causal dependence between the two time series. To address this issue we have considered the index $S(X \mid Y)$ that measures the nonlinear interdependency between two time series $X$ and $Y$, as described in [14]. More precisely, from the time series $\mathbf{x}$ and $\mathbf{y}$, one reconstructs delay vectors $\mathbf{x}_{n}=\left(x_{n}, \ldots, x_{n-(m-1) \tau}\right)$ and $\mathbf{y}_{n}$ $=\left(y_{n}, \ldots, y_{n-(m-1) \tau}\right)$, where $n=1, \ldots, N$ is the time index, $m$ is the embedding dimension, and $\tau$ denotes the time lag. Let $r_{n, j}$ and $s_{n, j}, j=1, \ldots, k$, denote the time indices of the $k$ nearest neighbors of $\mathbf{x}_{n}$ and $\mathbf{y}_{n}$, respectively. For each $\mathbf{x}_{n}$, the mean squared Euclidean distance to its $k$ neighbors is defined as

$$
R_{n}^{(k)}(\mathbf{X})=\frac{1}{k} \sum_{j=1}^{k}\left(\mathbf{x}_{n}-\mathbf{x}_{r_{n, j}}\right)^{2},
$$

while the $\mathbf{Y}$-conditioned mean squared Euclidean distance is defined by replacing the nearest neighbors by the equal time partners of the closest neighbors of $\mathbf{y}_{n}$,

$$
R_{n}^{(k)}(\mathbf{X} \mid \mathbf{Y})=\frac{1}{k} \sum_{j=1}^{k}\left(\mathbf{x}_{n}-\mathbf{x}_{s_{n, j}}\right)^{2}
$$

The interdependence measure is then defined as

$$
S(\mathbf{X} \mid \mathbf{Y})=\frac{1}{N} \sum_{n=1}^{N} \frac{R_{n}^{(k)}(\mathbf{X})}{R_{n}^{(k)}(\mathbf{X} \mid \mathbf{Y})}
$$

$S(X \mid Y)$ is an asymmetric quantity and the degree of asymmetry is connected to the causal relationship between the two time series, in other words if $S(X \mid Y)$ is much greater than $S(Y \mid X)$ then we may conclude that $Y$ is driving $X$. On the other hand each of these values measures by its size the degree of interdependency, $S=0 \quad(S=1)$ meaning minimal (maximal) interdependency. We evaluated these indexes both on the SAP and DAP time series $x_{S}(t), x_{D}(t)$ and on their phases $\theta_{S}(t), \theta_{D}(t)$. In both cases the asymmetry was not significant, which means that there is no causal relationship between the SAP and DAP time series; however, the results obtained with the phases are always much greater than those obtained with the full signals. Quantitatively, the average values for the $\mathrm{HF}$ band are $S\left(x_{D} \mid x_{S}\right)=4.8 \times 10^{-3}$ and $S\left(x_{S} \mid x_{D}\right)=4.6 \times 10^{-3}$, which shows a very small asymmetry and, at the same time, a very small interdependency. As to the phases, we get $S\left(\theta_{D} \mid \theta_{S}\right)=0.899$ and $S\left(\theta_{S} \mid \theta_{D}\right)=0.901$, 
which on the contrary shows a larger interdependency. Similar results are obtained in VLF and LF bands. Besides showing the absence of a causal relation, these results confirm that in these systems of oscillators the main source of information on the underlying structures resides in the dynamics of the phases.

\section{CONCLUSIONS}

Our results represent an original analysis of the relation between systolic/diastolic blood pressure, which completes previous studies [4]. The measured delays between the oscil- latory components of systolic and diastolic blood pressure time series show a change of sign going from low to high frequencies. We have addressed it within the paradigm of coupled nonlinear synchronous oscillators. We have shown, using Winfree and Kuramoto models, that once synchronization is achieved, the phase delay between oscillators is determined by the underlying structure and we have found a linear relationship between oscillator phase shifts and the difference of their natural frequencies. We then used these results to describe our findings that confirm that changes in the modulating factors of arterial pressure affect differently the systolic and diastolic pressure values [3].
[1] A. T. Winfree, The Geometry of Biological Time (Springer, New York, 1980).

[2] A. Malliani et al., Circulation 84, 482 (1991); J. K. Triedman and P. Saul, ibid. 89, 169 (1994); J. P. Saul, Am. J. Physiol. 261, H153 (1991).

[3] N. Stergiopulos et al., Am. J. Physiol. 270, 2050 (1996).

[4] G. D. Pinna, R. Maestri, M. T. La Rovere, and A. Mortara, Comput. Cardiol. 24, 207 (1997).

[5] A. T. Winfree, J. Theor. Biol. 16, 15 (1967).

[6] Y. Kuramoto, in International Symposium on Mathematical Problems in Theoretical Physics, Vol. 39 of Lecture Notes in Physics, edited by H. Araki (Springer-Verlag, Berlin, 1975); Chemical Oscillations, Waves and Turbulence (SpringerVerlag, Berlin, 1984).

[7] S. H. Strogatz, Physica D 143, 1 (2000).

[8] J. T. Ariaratnam and S. H. Strogatz, Phys. Rev. Lett. 86, 4278 (2001).
[9] T. J. Walker, Science 166, 891 (1969); E. Sismondo, ibid. 249, 55 (1990).

[10] J. Buck, Q. Rev. Biol. 63, 265 (1988).

[11] C. S. Peskin, Mathematical Aspects of Heart Physiology (Courant Institute of Mathematics and Science, New York, 1975); D. C. Michaels, E. P. Matyas, and J. Jalife, Circ. Res. 61, 704 (1987).

[12] The origin of this dependence on $\omega_{0}=2$ is in the first two terms of Eq. (6) that only vanish in the $N \rightarrow \infty, t \rightarrow \infty$ limit. As the relevance of these terms is regulated by the relative width of frequencies $\gamma / \omega_{0}$, a better agreement is obtained with $\omega_{0}=2$.

[13] P. Tass, M. G. Rosenblum, J. Weule, J. Kurths, A. Pikovsky, J. Volkmann, A. Schnitzler, and H. J. Freund, Phys. Rev. Lett. 81, 3291 (1998).

[14] J. Arnhold, P. Grassberger, K. Lehnertz, and C. E. Elger, Physica D 134, 419 (1999). 